\title{
Segal-Bargmann-Fock modules of monogenic functions
}

Cite as: J. Math. Phys. 58, 103507 (2017); https://doi.org/10.1063/1.5008651

Submitted: 07 September 2016 . Accepted: 09 October 2017 . Published Online: 23 October 2017

Dixan Peña Peña, Irene Sabadini, and Franciscus Sommen
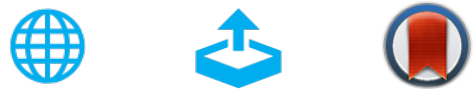

View Online

\section{ARTICLES YOU MAY BE INTERESTED IN}

From the discrete Weyl-Wigner formalism for symmetric ordering to a numberphase Wigner function

Journal of Mathematical Physics 58, 102106 (2017); https://

doi.org/10.1063/1.5008653

Coherent state transforms and the Weyl equation in Clifford analysis

Journal of Mathematical Physics 58, 013503 (2017); https://

doi.org/10.1063/1.4974449

Entropic uncertainty measures for large dimensional hydrogenic systems

Journal of Mathematical Physics 58, 103302 (2017); https://

doi.org/10.1063/1.5006569

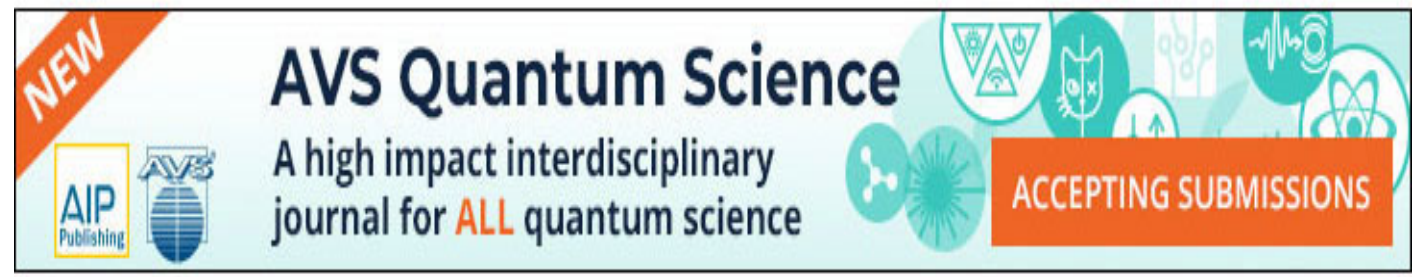




\title{
Segal-Bargmann-Fock modules of monogenic functions
}

\author{
Dixan Peña Peña, ${ }^{1, a)}$ Irene Sabadini, ${ }^{1, b)}$ and Franciscus Sommen ${ }^{2, c)}$ \\ ${ }^{1}$ Dipartimento di Matematica, Politecnico di Milano, Via E. Bonardi 9, 20133 Milano, Italy \\ ${ }^{2}$ Clifford Research Group, Department of Mathematical Analysis, Faculty of Engineering \\ and Architecture, Ghent University, Krijgslaan 281-S8, 9000 Gent, Belgium
}

(Received 7 September 2016; accepted 9 October 2017; published online 23 October 2017)

In this paper, we introduce the classical Segal-Bargmann transform starting from the basis of Hermite polynomials and extending it to Clifford algebra-valued functions. Then we apply the results to monogenic functions and prove that the Segal-Bargmann kernel corresponds to the kernel of the Fourier-Borel transform for monogenic functionals. This kernel is also the reproducing kernel for the monogenic Bargmann module. Published by AIP Publishing. https://doi.org/10.1063/1.5008651

\section{INTRODUCTION}

The Segal-Bargmann transform can be seen as a unitary map from spaces of square-integrable functions to spaces of holomorphic functions square-integrable with respect to a Gaussian density (see Refs. 1, 12, and 13). The latter class of functions is known in the literature under different names: Bargmann or Segal-Bargmann or Fock spaces either in one or several complex variables (see Refs. 6, 10, and 17). In this paper, we do not enter historical discussion on the nomenclature, and we will call them Segal-Bargmann-Fock spaces. These spaces are important in several different settings: in quantum mechanics, where they are used for the description of the spaces via tensor products; in infinite dimensional analysis and in free analysis, since these spaces are related to the white noise space (a probability space) and to the theory of stochastic distributions, see Ref. 16.

It is natural to consider another higher dimensional extension, namely, the one based on monogenic functions with values in a Clifford algebra. Recently, in Refs. 8 and 9, the authors have pointed out that this approach can be useful in studying quantum systems with internal, discrete degrees of freedom corresponding to nonzero spins.

Square-integrable holomorphic functions are well known in the literature, and they may belong to Hardy or Bergman spaces, whose reproducing kernels are rational functions. In the case of the Segal-Bargmann-Fock spaces, the kernel is an exponential function. This reproducing kernel is not bounded, and this fact makes some computations in this framework more complicated. On the other hand, the fact that Segal-Bargmann-Fock spaces are defined on the whole space (either $\mathbb{C}$ or $\mathbb{C}^{m}$ ) makes other techniques from Fourier analysis available. From the physics point of view, the (normalized) reproducing kernels of the Segal-Bargmann-Fock spaces are the so-called coherent states in quantum mechanics.

In this paper, we consider the higher dimensional framework based on monogenic functions. We can introduce a notion of Segal-Bargmann module (over the Clifford algebra) and of Segal-BargmannFock transform. The fact that we have a Fischer decomposition allows proving a relation between the projection of the transform onto its monogenic part and the Fourier-Borel kernel. Equipping the SegalBargmann-Fock module with the Fischer inner product, we can also show that the Segal-Bargmann transform is still an isometry on harmonic polynomials.

The plan of this paper is the following. In Sec. II, we revise the classical Segal-Bargmann transform. Then in Sec. III, we apply the results of Sec. II to the case of monogenic functions. In Sec. IV, we study monogenic Bargmann modules. Finally in Sec. V, we make summarizing remarks.

\footnotetext{
${ }^{a)}$ E-mail: dixanpena@gmail.com

b) E-mail: irene.sabadini@ polimi.it

c) E-mail: franciscus.sommen@ugent.be
} 


\section{THE CLASSICAL SEGAL-BARGMANN TRANSFORM}

The Segal-Bargmann transform is a very well-known operator acting from the space $L^{2}(\mathbb{R})$ and the Segal-Bargmann-Fock space which is unitary and allows identifying these two spaces (see Refs. 1, 12, and 13 and the recent monographs ${ }^{10,17}$ ).

The Segal-Bargmann-Fock space $\mathcal{B}(\mathbb{C})$ is the Hilbert space of entire functions which are squareintegrable with respect to the Gaussian density, i.e.,

$$
\frac{1}{\pi} \int_{\mathbb{C}} \exp \left(-|z|^{2}\right)|f(z)|^{2} d x d y<\infty .
$$

For any $f \in L^{2}(\mathbb{R})$, we define the Segal-Bargmann transform $\mathcal{B}: L^{2}(\mathbb{R}) \rightarrow \mathcal{B}(\mathbb{C})$ (sometimes called the Segal-Bergmann-Fock transform) as

$$
\mathcal{B}[f](z)=\frac{1}{\sqrt{2 \pi}} \int_{\mathbb{R}} \exp \left(-\frac{z^{2}}{2}+x z-\frac{x^{2}}{4}\right) f(x) d x,
$$

for any $f \in L^{2}(\mathbb{R})$.

Let us consider in $L^{2}(\mathbb{R})$, the basis $\left\{\psi_{k}(x)\right\}$, where

$$
\psi_{k}(x)=H_{k}(x) e^{-x^{2} / 4}, \quad H_{k}(x)=(-1)^{k} e^{x^{2} / 2} \partial_{x}^{k} e^{-x^{2} / 2} .
$$

Note that $H_{k}(x)$ are the well-known Hermite polynomials. This basis is orthogonal, in fact,

$$
\left\langle\psi_{\ell}, \psi_{k}\right\rangle=\frac{1}{\sqrt{2 \pi}} \int_{\mathbb{R}} \overline{\psi_{\ell}(x)} \psi_{k}(x) d x=\delta_{\ell k} k !,
$$

where $\delta_{\ell k}$ is the Kronecker delta.

Using integration by parts, we obtain

$$
\begin{aligned}
\mathcal{B}\left[\psi_{k}\right](z) & =\frac{1}{\sqrt{2 \pi}} \int_{\mathbb{R}} \exp \left(-\frac{(z-x)^{2}}{2}\right) H_{k}(x) d x \\
& =\frac{1}{\sqrt{2 \pi}} \int_{\mathbb{R}} \partial_{x}^{k}\left(\exp \left(-\frac{z^{2}}{2}+x z\right)\right) \exp \left(-x^{2} / 2\right) d x \\
& =\frac{1}{\sqrt{2 \pi}} z^{k} \int_{\mathbb{R}} \exp \left(-\frac{(z-x)^{2}}{2}\right) d x \\
& =z^{k} .
\end{aligned}
$$

Let us consider the so-called Fischer space, namely, the space of real analytic functions equipped with the inner product

$$
[R, S]=\left.\overline{R\left(\partial_{x}\right)} S(x)\right|_{x=0} .
$$

To consider functions defined over the real numbers, it is necessary to obtain a match between the Fischer and the Bargmann inner products.

If we consider instead of the complex variable $z$ a real variable $u$, we can use the Fischer inner product to get the equality $\left[u^{\ell}, u^{k}\right]=\delta_{\ell k} k$ !.

The above computations show that the Segal-Bargmann-Fock transform $f(x) \mapsto \mathcal{B}[f](u)$ is an isometry between $L^{2}(\mathbb{R})$ and the Fischer space.

On the other hand, we can consider the space $\mathcal{O}(\mathbb{C})$ of entire holomorphic functions equipped with the inner product

$$
\langle f, g\rangle=\frac{1}{\pi} \int_{\mathbb{C}} \exp \left(-|z|^{2}\right) \overline{f(z)} g(z) d x d y, \quad z=x+i y .
$$

We note that the basis $\left\{z^{k}\right\}$ is orthogonal, in fact,

$$
\left\langle z^{\ell}, z^{k}\right\rangle=\frac{1}{\pi} \int_{\mathbb{C}} \exp \left(-|z|^{2}\right) \bar{z}^{k} z^{\ell} d x d y=0 \quad \text { for } k \neq \ell .
$$

Moreover

$$
\left\langle z^{k}, z^{k}\right\rangle=\frac{1}{\pi} \int_{0}^{2 \pi} \int_{0}^{\infty} \exp \left(-r^{2}\right) r^{2 k} r d r d \theta=k !, \quad \text { where } z=r e^{\theta} .
$$


Then we have that $\mathcal{B}: L^{2}(\mathbb{R}) \rightarrow \mathcal{B}(\mathbb{C})$ is an isometry. Indeed, the Fischer inner product for functions defined in $\mathbb{R}$ is equal to the Bargmann inner product of the holomorphic extensions.

This discussion can be extended from one to several complex variables.

Definition 2.1. The Segal-Bargmann-Fock space $\mathcal{B}\left(\mathbb{C}^{m}\right)$ is defined as the Hilbert space of entire functions $f$ in $\mathbb{C}^{m}$ which are square-integrable with respect to the $2 m$-dimensional Gaussian density, i.e.,

$$
\frac{1}{\pi^{m}} \int_{\mathbb{C}^{m}} \exp \left(-|\underline{z}|^{2}\right)|f(\underline{z})|^{2} d \underline{x} d \underline{y}<\infty, \quad \underline{z}=\underline{x}+i \underline{y}
$$

and equipped with the inner product

$$
\langle f, g\rangle=\frac{1}{\pi^{m}} \int_{\mathbb{C}^{m}} \exp \left(-|\underline{z}|^{2}\right) \overline{f(\underline{z})} g(\underline{z}) d \underline{x} d \underline{y} .
$$

Let us denote by $\underline{x}$ the $m$-tuple $\left(x_{1}, \ldots, x_{m}\right) \in \mathbb{R}^{m}$. We consider the space $L^{2}\left(\mathbb{R}^{m}\right)$ with the basis $\left\{\psi_{k_{1} \ldots k_{m}}(\underline{x})\right\}$, where

$$
\psi_{k_{1} \ldots k_{m}}(\underline{x})=H_{k_{1} \ldots k_{m}}(\underline{x}) e^{-|\underline{x}|^{2} / 4}
$$

and $H_{k_{1} \ldots k_{m}}$ are the Hermite polynomials in $\mathbb{R}^{m}$ given by

$$
H_{k_{1} \ldots k_{m}}(\underline{x}) e^{-|\underline{x}|^{2} / 2}=(-1)^{k_{1}+\cdots+k_{m}} \partial_{x_{1}}^{k_{1}} \ldots \partial_{x_{m}}^{k_{m}} e^{-|\underline{x}|^{2} / 2} .
$$

Definition 2.2. The Segal-Bargmann-Fock transform $\mathcal{B}: L^{2}\left(\mathbb{R}^{m}\right) \rightarrow \mathcal{B}\left(\mathbb{C}^{m}\right)$ is defined by

$$
\mathcal{B}[f](\underline{z})=\frac{1}{(2 \pi)^{m / 2}} \int_{\mathbb{R}^{m}} \exp \left(-\frac{\underline{z} \cdot \underline{z}}{2}+\underline{x} \cdot \underline{z}-\frac{\underline{x} \cdot \underline{x}}{4}\right) f(\underline{x}) d \underline{x}, \quad \underline{x} \cdot \underline{z}=\sum_{j=1}^{m} x_{j} z_{j},
$$

for any $f \in L^{2}\left(\mathbb{R}^{m}\right)$.

Remark 2.3. Similar to the one variable case, we have that

$$
\mathcal{B}\left[\psi_{k_{1} \ldots k_{m}}\right](\underline{z})=z_{1}^{k_{1}} \ldots z_{m}^{k_{m}} .
$$

Finally note that $\left\|\psi_{k_{1} \ldots k_{m}}\right\|^{2}=k_{1} ! \ldots k_{m}$ !. It follows from this that $\mathcal{B}: L^{2}\left(\mathbb{R}^{m}\right) \rightarrow \mathcal{B}\left(\mathbb{C}^{m}\right)$ is an isometry.

\section{THE MONOGENIC CASE}

Let us denote by $\mathbb{R}_{m}$ the real Clifford algebra generated by $m$ imaginary units $e_{1}, \ldots, e_{m}$. The multiplication in this associative algebra is determined by the relations

$$
e_{j} e_{k}+e_{k} e_{j}=-2 \delta_{i j}
$$

An element $a \in \mathbb{R}_{m}$ can be written as

$$
a=\sum_{A} a_{A} e_{A}, \quad x_{A} \in \mathbb{R}
$$

where the basis elements $e_{A}=e_{j_{1}} \ldots e_{j_{k}}$ are defined for every subset $A=\left\{j_{1}, \ldots, j_{k}\right\}$ of $\{1, \ldots, m\}$ with $j_{1}<\cdots<j_{k}$. For the empty set, one puts $e_{\emptyset}=1$, the latter being the identity element.

Observe that the dimension of $\mathbb{R}_{m}$ as a real linear space is $2^{m}$. Furthermore, conjugation in $\mathbb{R}_{m}$ is given by $\bar{a}=\sum_{A} a_{A} \bar{e}_{A}$, where $\bar{e}_{A}=\bar{e}_{j_{k}} \ldots \bar{e}_{j_{1}}$ with $\bar{e}_{j}=-e_{j}, j=1, \ldots, m$.

In the Clifford algebra $\mathbb{R}_{m}$, we can identify the so-called 1-vectors, namely, the linear combinations with real coefficients of the elements $e_{j}, j=1, \ldots, m$, with the vectors in the Euclidean space $\mathbb{R}^{m}$. The correspondence is given by the map $\left(x_{1}, \ldots, x_{m}\right) \mapsto \underline{x}=x_{1} e_{1}+\cdots+x_{m} e_{m}$ and it is obviously one-to-one.

The norm of a 1-vector $\underline{x}$ is defined as $|\underline{x}|=\sqrt{x_{1}^{2}+\cdots+x_{m}^{2}}$ and clearly $\underline{x}^{2}=-|\underline{x}|^{2}$. The product of two 1-vectors $\underline{x}=\sum_{j=1}^{m} x_{j} e_{j}$ and $\underline{y}=\sum_{j=1}^{m} y_{j} e_{j}$ splits into a scalar part and a 2-vector or the so-called bivector part

$$
\underline{x} \underline{y}=-\langle\underline{x}, \underline{y}\rangle+\underline{x} \wedge \underline{y}
$$


where

$$
\begin{aligned}
& \langle\underline{x}, \underline{y}\rangle=\underline{x} \cdot \underline{y}=-\frac{1}{2}(\underline{x} \underline{y}+\underline{y} \underline{x})=\sum_{j=1}^{m} x_{j} y_{j}, \\
& \underline{x} \wedge \underline{y}=\frac{1}{2}(\underline{x} \underline{y}-\underline{y} \underline{x})=\sum_{j=1}^{m} \sum_{k=j+1}^{m}\left(x_{j} y_{k}-x_{k} y_{j}\right) e_{j} e_{k} .
\end{aligned}
$$

The complex Clifford algebra $\mathbb{C}_{m}$ can be seen as the complexification of the real Clifford algebra $\mathbb{R}_{m}$, i.e., $\mathbb{C}_{m}=\mathbb{R}_{m} \oplus i \mathbb{R}_{m}$. Any complex Clifford number $c \in \mathbb{C}_{m}$ may be written as $c=a+i b, a, b \in \mathbb{R}_{m}$, leading to the definition of the Hermitian conjugation: $c^{\dagger}=\bar{a}-i \bar{b}$.

The first order differential operator

$$
\partial_{\underline{x}}=\sum_{j=1}^{m} e_{j} \partial_{x_{j}}
$$

is called the Dirac operator in $\mathbb{R}^{m}$. Functions in the kernel of this operator are known as monogenic functions (see, e.g., Refs. 2-4 and 7).

Definition 3.1. A function $f: \Omega \subset \mathbb{R}^{m} \rightarrow \mathbb{C}_{m}$ defined and continuously differentiable in the open set $\Omega$ is said to be (left) monogenic if $\partial_{\underline{x}} f(\underline{x})=0$ in $\Omega$. We denote by $\mathcal{M}\left(\mathbb{R}^{m}\right)$ the right $\mathbb{C}_{m}$-module of monogenic functions in $\mathbb{R}^{m}$.

A basic result in Clifford analysis is the so-called Fischer decomposition. Every homogeneous polynomial $R_{k}$ of degree $k$ can be uniquely decomposed as

$$
R_{k}(\underline{x})=M_{k}(\underline{x})+\underline{x} R_{k-1}(\underline{x}),
$$

where $M_{k}$ and $R_{k-1}$ are homogeneous polynomials and $M_{k} \in \mathcal{M}\left(\mathbb{R}^{m}\right)$. The monogenic polynomial $M_{k}$ is called the monogenic part of $R_{k}$ denoted by $\mathcal{M}\left(R_{k}\right)$.

If $f$ is real analytic function near the origin, then it admits a decomposition of the form $f(\underline{x})=\sum_{k=0}^{\infty} R_{k}(\underline{x})$ in an open ball centred at the origin. The monogenic part of $f$ is thus defined by

$$
\mathcal{M}(f(\underline{x}))=\sum_{k=0}^{\infty} \mathcal{M}\left(R_{k}(\underline{x})\right) .
$$

Let us recall the definition of the Clifford-Hermite polynomials $H_{s, k}(\underline{x})$. They are polynomials with real coefficients in $\underline{x}$ of degree $s$ satisfying

$$
H_{s, k}(\underline{x}) e^{-|\underline{x}|^{2} / 2} P_{k}(\underline{x})=(-1)^{s} \partial_{\underline{x}}^{S}\left(P_{k}(\underline{x}) e^{-|\underline{x}|^{2} / 2}\right),
$$

where $P_{k}(\underline{x})$ denotes a homogeneous polynomial of degree $k$ in $\mathcal{M}\left(\mathbb{R}^{m}\right)$.

Put $\psi_{s, k}(\underline{x})=H_{s, k}(\underline{x}) e^{-|\underline{x}|^{2} / 4}$. The set of functions $\left\{\psi_{s, k}(x) P_{k}(\underline{x}): s, k \in \mathbb{N}\right\}$ is an orthogonal basis for $L^{2}\left(\mathbb{R}^{m}\right)$ (see Ref. 14). Recalling Definition 2.2, we have the following:

Theorem 3.2. Assume that $P_{k}(\underline{x})$ is a homogeneous polynomial of degree $k$ in $\mathcal{M}\left(\mathbb{R}^{m}\right)$. Then the following formula holds:

$$
\mathcal{B}\left[\psi_{s, k}(\underline{x}) P_{k}(\underline{x})\right](\underline{z})=\underline{z}^{s} \mathcal{B}\left[P_{k}(\underline{x}) e^{-|\underline{x}|^{2} / 4}\right](\underline{z}) .
$$

Proof. The formula follows using integration by parts in higher dimensions,

$$
\begin{aligned}
\mathcal{B}\left[\psi_{s, k}(\underline{x}) P_{k}(\underline{x})\right](\underline{z}) & =\frac{1}{(2 \pi)^{m / 2}} \int_{\mathbb{R}^{m}} \exp \left(-\frac{\underline{z} \cdot \underline{z}}{2}+\underline{x} \cdot \underline{z}-\frac{\underline{x} \cdot \underline{x}}{4}\right) \psi_{s, k}(\underline{x}) P_{k}(\underline{x}) d \underline{x} \\
& =\frac{(-1)^{s}}{(2 \pi)^{m / 2}} \int_{\mathbb{R}^{m}} \exp \left(-\frac{\underline{z} \cdot \underline{z}}{2}+\underline{x} \cdot \underline{z}\right) \partial_{\underline{x}}^{s}\left(P_{k}(\underline{x}) e^{-|\underline{x}|^{2} / 2}\right) d \underline{x} \\
& =\frac{1}{(2 \pi)^{m / 2}} \int_{\mathbb{R}^{m}} \partial_{\underline{x}}^{s}\left(\exp \left(-\frac{\underline{z} \cdot \underline{z}}{2}+\underline{x} \cdot \underline{z}\right)\right) P_{k}(\underline{x}) e^{-|\underline{x}|^{2} / 2} d \underline{x} \\
& =\underline{z}^{s} \mathcal{B}\left[P_{k}(\underline{x}) e^{-|\underline{x}|^{2} / 4}\right](\underline{z}) .
\end{aligned}
$$


The next problem is to compute $\mathcal{B}\left[P_{k}(\underline{x}) e^{-|\underline{x}|^{2} / 4}\right]$. To this end, we consider an example from which the general result will follow.

Lemma 3.3. Let $P_{k}(\underline{x})=\left(x_{1}-e_{1} e_{2} x_{2}\right)^{k}$. Then

$$
\mathcal{B}\left[P_{k}(\underline{x}) e^{-|\underline{x}|^{2} / 4}\right](\underline{z})=\left(z_{1}-e_{1} e_{2} z_{2}\right)^{k}=P_{k}(\underline{z}) .
$$

Proof. Let $Z=x_{1}-e_{1} e_{2} x_{2}$ and $2 \partial_{\bar{Z}}=\partial_{x_{1}}-e_{1} e_{2} \partial_{x_{2}}$. Note that

$$
e^{-|\underline{x}|^{2} / 2}=\exp \left(-\frac{1}{2} Z \bar{Z}\right) \exp \left(\sum_{j=3}^{m}-\frac{1}{2} x_{j}^{2}\right) .
$$

Thus we have

$$
\begin{aligned}
Z^{k} e^{-|x|^{2} / 2} & =\left(\left(-2 \partial_{\bar{Z}}\right)^{k} \exp \left(-\frac{1}{2} Z \bar{Z}\right)\right) \exp \left(\sum_{j=3}^{m}-\frac{1}{2} x_{j}^{2}\right) \\
& =\left(-\left(\partial_{x_{1}}-e_{1} e_{2} \partial_{x_{2}}\right)\right)^{k} e^{-|\underline{x}|^{2} / 2} .
\end{aligned}
$$

Therefore

$$
\begin{aligned}
\mathcal{B}\left[P_{k}(\underline{x}) e^{-|\underline{x}|^{2} / 4}\right](\underline{z}) & =\frac{1}{(2 \pi)^{m / 2}} \int_{\mathbb{R}^{m}}\left(\left(\partial_{x_{1}}-e_{1} e_{2} \partial_{x_{2}}\right)^{k} \exp \left(-\frac{\underline{z} \cdot \underline{z}}{2}+\underline{x} \cdot \underline{z}\right)\right) e^{-|\underline{x}|^{2} / 2} d \underline{x} \\
& =\frac{\left(z_{1}-e_{1} e_{2} z_{2}\right)^{k}}{(2 \pi)^{m / 2}} \int_{\mathbb{R}^{m}} \exp \left(-\frac{(\underline{z}-\underline{x}) \cdot(\underline{z}-\underline{x})}{2}\right) d \underline{x} \\
& =\frac{\left(z_{1}-e_{1} e_{2} z_{2}\right)^{k}}{(2 \pi)^{m / 2}} \int_{\mathbb{R}^{m}} \exp \left(-\frac{|\underline{x}|^{2}}{2}\right) d \underline{x} \\
& =\left(z_{1}-e_{1} e_{2} z_{2}\right)^{k} .
\end{aligned}
$$

We can now prove the following simple but important result:

Theorem 3.4. Suppose that $P_{k}(\underline{x})$ is a homogeneous polynomial of degree $k$ in $\mathcal{M}\left(\mathbb{R}^{m}\right)$. Then the following formula holds:

$$
\mathcal{B}\left[H_{s, k}(\underline{x}) e^{-|\underline{x}|^{2} / 4} P_{k}(\underline{x})\right](\underline{z})=\underline{z}^{s} P_{k}(\underline{z}) .
$$

Proof. We show that the formula that we have established for $P_{k}(\underline{x})=\left(x_{1}-e_{1} e_{2} x_{2}\right)^{k}$ in Lemma 3.3 holds for a general $P_{k}(\underline{x})$. First of all, due to the $\operatorname{spin}(m)$-invariance, the result also holds for monogenic plane wave functions of the form

$$
(\langle\underline{x}, \underline{t}\rangle-\underline{t} \underline{s}\langle\underline{x}, \underline{s}\rangle)^{k},
$$

where $\underline{t}$ and $\underline{s}$ are orthogonal unit vectors. Then we notice that the space of homogeneous monogenic polynomials of degree $k$ is spanned by finitely many monogenic plane waves $(\langle\underline{x}, \underline{t}\rangle-\underline{t} \underline{s}\langle\underline{x}, \underline{s}\rangle)^{k}$ for some choices of the parameters $(\underline{t}, \underline{s})$. This, combined with Theorem 3.2, proves the result.

\section{MONOGENIC BARGMANN MODULES}

We will use the following notations.

Definition 4.1. Let $s \in \mathbb{N}$. We denote by $\mathcal{M}^{s}\left(\mathbb{R}^{m}\right)$ the right $\mathbb{C}_{m}$-module of s-monogenic functions on $\mathbb{R}^{m}$, namely, the set of smooth functions in the kernel of $\partial_{x}^{s}$. Next, by $\mathcal{M}^{s}\left(\mathbb{C}^{m}\right)$, we denote the right $\mathbb{C}_{m}$-module of s-monogenic functions on $\mathbb{C}^{m}$, namely, the set of entire holomorphic functions in the kernel of $\partial_{\underline{z}}^{s}$, where $\partial_{\underline{z}}=\sum_{j=1}^{m} e_{j} \partial_{z_{j}}$ is the complexified Dirac operator.

Note that $\mathcal{M}{ }^{s}\left(\mathbb{C}^{m}\right)$ is spanned by the set of polynomials of the form $\underline{z}^{j} P_{k}(\underline{z}), j=0, \ldots, s-1$, where $P_{k}(\underline{z})$ is the complex spherical monogenics of degree $k \in \mathbb{N}$.

Let us now define the $s$-monogenic Bargmann modules. 
Definition 4.2. For any $s \in \mathbb{N}$, the s-monogenic Bargmann module $\mathcal{M} \mathcal{B}^{s}\left(\mathbb{C}^{m}\right)$ is defined as

$$
\mathcal{M} \mathcal{B}^{s}\left(\mathbb{C}^{m}\right)=\mathcal{M}^{s}\left(\mathbb{C}^{m}\right) \cap \mathcal{B}\left(\mathbb{C}^{m}\right),
$$

and it is is equipped with the inner product defined in $\mathcal{B}\left(\mathbb{C}^{m}\right)$.

The Segal-Bargmann transform may act on $s$-monogenic functions as described in the following:

Proposition 4.3. The map

$$
\mathcal{B}: \mathcal{M}^{s}\left(\mathbb{R}^{m}\right) e^{-|\underline{x}|^{2} / 4} \cap L^{2}\left(\mathbb{R}^{m}\right) \rightarrow \mathcal{M} \mathcal{B}^{s}\left(\mathbb{C}^{m}\right)
$$

is an isometry (and it is even unitary).

Proof. The proof follows the standard arguments in the complex case and since $H_{s, k}(\underline{x}) P_{k}(\underline{x})$, $j=0, \ldots, s-1, \operatorname{span} \mathcal{M}^{s}\left(\mathbb{R}^{m}\right)$, it is a consequence of the previous Theorem 3.4.

Remark 4.4. From the Fischer decomposition we obtain

$$
\frac{\langle\underline{x}, \underline{u}\rangle^{k}}{k !}=\sum_{s=0}^{k} \underline{x}^{s} Z_{k, s}(\underline{x}, \underline{u}) \underline{u}^{s},
$$

where $Z_{k, s}(\underline{x}, \underline{u})$ are the so-called zonal spherical monogenics (see Ref. 15). The latter are homogeneous polynomials of degree $k-s$ in $\underline{x}$ and $\underline{u}$ and satisfy the two-sided biregular system $\partial_{\underline{x}} Z_{k, s}(\underline{x}, \underline{u})=Z_{k, s}(\underline{x}, \underline{u}) \partial_{\underline{u}}=0$.

One can also check that

$$
Z_{k, s}(\underline{x}, \underline{u})=\frac{Z_{k-s, 0}(\underline{x}, \underline{u})}{\beta_{s, k-s} \ldots \beta_{1, k-s}}, \quad k \geq s
$$

with $\beta_{2 s, k}=-2 s, \beta_{2 s+1, k}=-(2 s+2 k+m)$. Moreover,

$$
\begin{aligned}
Z_{k}(\underline{x}, \underline{u})=Z_{k, 0}(\underline{x}, \underline{u}) & \Gamma\left(\frac{m}{2}-1\right) \\
& =\frac{2^{k+1} \Gamma\left(k+\frac{m}{2}\right)}{2^{2}}(|\underline{x}||\underline{u}|)^{k}\left[(k+m-2) C_{k}^{\frac{m}{2}-1}(t)+(m-2) \frac{\underline{x} \wedge \underline{u}}{|\underline{x}||\underline{u}|} C_{k-1}^{\frac{m}{2}}(t)\right],
\end{aligned}
$$

where $C_{k}^{\alpha}(t)$ denotes the classical Gegenbauer polynomial and $t=\frac{\langle\underline{x}, \underline{u}\rangle}{|\underline{x}||\underline{u}|}$.

Remark 4.5. Therefore, the Fischer decomposition of $e^{\langle\underline{x}, \underline{u}\rangle}$ has the form

$$
e^{\langle\underline{x}, \underline{u}\rangle}=\sum_{k=0}^{\infty} \frac{\langle\underline{x}, \underline{u}\rangle^{k}}{k !}=\sum_{k=0}^{\infty} \sum_{s=0}^{k} \underline{x}^{s} Z_{k, s}(\underline{x}, \underline{u}) \underline{u}^{s}=\sum_{s=0}^{\infty} \underline{x}^{s} E_{s}(\underline{x}, \underline{u}) \underline{u}^{s},
$$

with $E_{s}(\underline{x}, \underline{u})=\sum_{k=s}^{\infty} Z_{k, s}(\underline{x}, \underline{u})$.

We note that $E(\underline{x}, \underline{u})=E_{0}(\underline{x}, \underline{u})$ is the monogenic part of $e^{\langle\underline{x}, \underline{u}\rangle}$, which is known as the FourierBorel kernel. This function has been computed in a closed form using Bessel functions in Ref. 5 (see also Ref. 11).

Let us again denote by $\mathcal{M}$ the projection of a function onto its monogenic part in the complex monogenic Fischer decomposition.

Proposition 4.6. Let $f(\underline{x})=g(\underline{x}) e^{-|\underline{x}|^{2} / 4} \in L^{2}\left(\mathbb{R}^{m}\right)$. Then we have

$$
\mathcal{M}(\mathcal{B}[f](\underline{z}))=\frac{1}{(2 \pi)^{m / 2}} \int_{\mathbb{R}^{m}} E(\underline{z}, \underline{x}) g(\underline{x}) e^{-|\underline{x}|^{2} / 2} d \underline{x}=\left[E(\underline{z}, \underline{x})^{\dagger}, g(\underline{x})\right],
$$

where $[\cdot, \cdot]$ denotes the Fischer inner product. In particular when $g$ is monogenic, we obtain $\mathcal{M}(\mathcal{B}[f](\underline{z}))=g(\underline{z})$. 
Proof. From the above remarks, we obtain

$$
\mathcal{M}\left(\exp \left(-\frac{\underline{z} \cdot \underline{z}}{2}+\underline{x} \cdot \underline{z}-\frac{\underline{x} \cdot \underline{x}}{4}\right)\right)=\mathcal{M}(\exp (\underline{x} \cdot \underline{z})) e^{-|\underline{x}|^{2} / 4}=E(\underline{z}, \underline{x}) e^{-|\underline{x}|^{2} / 4} .
$$

The statement follows using

$$
\mathcal{M}(\mathcal{B}[f](\underline{z}))=\frac{1}{(2 \pi)^{m / 2}} \int_{\mathbb{R}^{m}} \mathcal{M}\left(\exp \left(-\frac{\underline{z} \cdot \underline{z}}{2}+\underline{x} \cdot \underline{z}-\frac{\underline{x} \cdot \underline{x}}{4}\right)\right) f(\underline{x}) d \underline{x} .
$$

\section{REAL $s$-MONOGENIC BARGMANN MODULES}

Let us recall that for harmonic polynomials $R(\underline{x}), S(\underline{x})$, we have (see also Ref. 7)

$$
[R(\underline{x}), S(\underline{x})]=\left.R\left(\partial_{\underline{x}}\right)^{\dagger} S(\underline{x})\right|_{\underline{x}=\underline{0}}=\frac{1}{(2 \pi)^{m / 2}} \int_{\mathbb{R}^{m}} R(\underline{x})^{\dagger} S(\underline{x}) e^{-|\underline{x}|^{2} / 2} d \underline{x} .
$$

For $P_{k}(\underline{x}) \in \mathcal{M}\left(\mathbb{R}^{m}\right)$, we have

$$
P_{k}(\underline{u})=\left[Z_{k}(\underline{u}, \underline{x})^{\dagger}, P_{k}(\underline{x})\right]=\left[E(\underline{u}, \underline{x})^{\dagger}, P_{k}(\underline{x})\right] .
$$

From this formula, one also obtains in another way that for every monogenic $g(\underline{x})$ such that $g(\underline{x}) e^{-|\underline{x}|^{2} / 4} \in L^{2}\left(\mathbb{R}^{m}\right)$, the following formula holds:

$$
g(\underline{u})=\frac{1}{(2 \pi)^{m / 2}} \int_{\mathbb{R}^{m}} E(\underline{u}, \underline{x}) g(\underline{x}) e^{-|\underline{x}|^{2} / 2} d \underline{x} .
$$

For general polynomials $R(\underline{x}), S(\underline{x})$, this link with the Fischer inner product no longer holds. However, we have that

$$
[R(\underline{x}), S(\underline{x})]=\frac{1}{\pi^{m}} \int_{\mathbb{C}^{m}} R(\underline{z})^{\dagger} S(\underline{z}) e^{-|\underline{z}|^{2}} d \underline{z} .
$$

For instance, take $R, S$ of the form $z_{1}^{k_{1}} \ldots z_{m}^{k_{m}}$. Moreover, $\mathcal{B}$ is an isometry between $L^{2}\left(\mathbb{R}^{m}\right)$ and the Segal-Bargmann-Fock module equipped with the above inner product. In particular, if $g \in \mathcal{M}^{s}\left(\mathbb{R}^{m}\right)$, we have that the map

$$
\mathcal{B}: g(\underline{x}) e^{-|\underline{x}|^{2} / 4} \mapsto h(\underline{z})=\mathcal{B}\left(g(\underline{x}) e^{-|\underline{x}|^{2} / 4}\right)
$$

is an isometry. Note also that $h$ is $s$-monogenic, but it is no longer true that $h(\underline{u})=g(\underline{u})$ for $\underline{u} \in \mathbb{R}^{m}$.

Let us consider $f \in \mathcal{B}\left(\mathbb{C}^{m}\right)$ and let $\mathcal{M}_{s}$ be the orthogonal projection onto the submodule of $s$-monogenic functions $\mathcal{M B} \mathcal{B}^{s}\left(\mathbb{C}^{m}\right)$. To be more precise, if

$$
f(\underline{z})=\sum_{j=0}^{\infty} \underline{z}^{j} f_{j}(\underline{z}), \quad \partial_{\underline{z}} f_{j}(\underline{z})=0,
$$

is the Fischer decomposition of $f$, then $\mathcal{M}_{s}(f(\underline{z}))=\sum_{j=0}^{s-1} \underline{z}_{j}^{j} f_{j}(\underline{z})$.

Define the function

$$
B_{s}(\underline{z}, \underline{x})=\mathcal{M}_{s}\left(\exp \left(-\frac{\underline{z} \cdot \underline{z}}{2}+\underline{x} \cdot \underline{z}\right)\right) .
$$

Theorem 5.1. The following formula holds:

$$
B_{s}(\underline{z}, \underline{x})=\sum_{j=0}^{s-1} \underline{z}^{j} \sum_{\ell=0}^{[j / 2]} \frac{1}{2^{\ell} \ell !} E_{j-2 \ell}(\underline{z}, \underline{x}) \underline{x}^{j-2 \ell} .
$$

Proof. We have that

$$
\exp \left(-\frac{\underline{z} \cdot \underline{z}}{2}\right)=\sum_{\ell=0}^{\infty} \frac{\underline{z}^{2 \ell}}{2^{\ell} \ell !}
$$

and

$$
\exp (\underline{x} \cdot \underline{z})=\sum_{k=0}^{\infty} \underline{z}^{k} E_{k}(\underline{z}, \underline{x}) \underline{x}^{k}
$$


Therefore

$$
\begin{aligned}
\exp \left(-\frac{\underline{z} \cdot \underline{z}}{2}+\underline{x} \cdot \underline{z}\right) & =\sum_{k, \ell=0}^{\infty} \frac{1}{2^{\ell} \ell !} \underline{z}^{k+2 \ell} E_{k}(\underline{z}, \underline{x}) \underline{x}^{k} \\
& =\sum_{j=0}^{\infty} \underline{z}^{j} \sum_{\ell=0}^{[j / 2]} \frac{1}{2^{\ell} \ell !} E_{j-2 \ell}(\underline{z}, \underline{x}) \underline{x}^{j-2 \ell}
\end{aligned}
$$

from which the desired result easily follows.

Remark 5.2. Note also the formula

$$
\mathcal{M}_{s}\left(\mathcal{B}\left[g(\underline{x}) e^{-|\underline{x}|^{2} / 4}\right](\underline{z})\right)=\frac{1}{(2 \pi)^{m / 2}} \int_{\mathbb{R}^{m}} \mathcal{M}_{s}\left(\exp \left(-\frac{\underline{z} \cdot \underline{z}}{2}+\underline{x} \cdot \underline{z}\right)\right) g(\underline{x}) e^{-|\underline{x}|^{2} / 2} d \underline{x} .
$$

Next, one may introduce the real s-monogenic Bargmann module, which is the set of $g \in \mathcal{M}^{s}\left(\mathbb{R}^{m}\right)$ such that $g(\underline{x}) e^{-|\underline{x}|^{2} / 4}$ is square-integrable.

Definition 5.3. For any $s \in \mathbb{N}$, the real s-monogenic Bargmann module $\mathcal{M} \mathcal{B}^{s}\left(\mathbb{R}^{m}\right)$ is defined by

$$
\mathcal{M} \mathcal{B}^{s}\left(\mathbb{R}^{m}\right)=\mathcal{M}^{s}\left(\mathbb{R}^{m}\right) \cap L^{2}\left(\mathbb{R}^{m}, e^{-|\underline{x}|^{2} / 2}\right) .
$$

It is equipped with the inner product in $L^{2}\left(\mathbb{R}^{m}, e^{-|\underline{x}|^{2} / 2}\right)$,

$$
\langle f, g\rangle=\frac{1}{(2 \pi)^{m / 2}} \int_{\mathbb{R}^{m}} f(\underline{x})^{\dagger} g(\underline{x}) e^{-|\underline{x}|^{2} / 2} d \underline{x} .
$$

As an additional remark, we note that for any $f \in \mathcal{M} \mathcal{B}^{s}\left(\mathbb{R}^{m}\right)$, we can define its Weierstrass transform $\mathcal{W}: \mathcal{M} \mathcal{B}^{s}\left(\mathbb{R}^{m}\right) \rightarrow \mathcal{M B} \mathcal{B}^{s}\left(\mathbb{C}^{m}\right)$ by

$$
\mathcal{W}[f](\underline{z})=\frac{1}{(2 \pi)^{m / 2}} \int_{\mathbb{R}^{m}} \exp \left(-\frac{(\underline{z}-\underline{x}) \cdot(\underline{z}-\underline{x})}{2}\right) f(\underline{x}) d \underline{x}=\mathcal{B}\left[f(\underline{x}) e^{-|\underline{x}|^{2} / 4}\right](\underline{z}) .
$$

With standard techniques, one can show that the map $\mathcal{W}: \mathcal{M} \mathcal{B}^{s}\left(\mathbb{R}^{m}\right) \rightarrow \mathcal{M} \mathcal{B}^{s}\left(\mathbb{C}^{m}\right)$ is an isometry, moreover

$$
\mathcal{W}[f](\underline{z})=\frac{1}{(2 \pi)^{m / 2}} \int_{\mathbb{R}^{m}} B_{s}(\underline{z}, \underline{x}) f(\underline{x}) e^{-|\underline{x}|^{2} / 2} d \underline{x} .
$$

\section{ACKNOWLEDGMENTS}

D. Peña Peña acknowledges the support of a Postdoctoral Fellowship given by Istituto Nazionale di Alta Matematica (INdAM) and cofunded by Marie Curie actions.

${ }^{1}$ Bargmann, V., "On a Hilbert space of analytic functions and an associated integral transform," Commun. Pure Appl. Math. 14, 187-214 (1961).

${ }^{2}$ Brackx, F., Delanghe, R., and Sommen, F., "Clifford analysis," in Pitman Research Notes in Mathematics series (Pitman Advanced Publishing Program, 1982), Vol. 76.

${ }^{3}$ Colombo, F., Sabadini, I., Sommen, F., and Struppa, D. C., Analysis of Dirac Systems and Computational Algebra, Volume 39 of Progress in Mathematical Physics (Birkhäuser, Boston, 2004).

${ }^{4}$ Delanghe, R., Sommen, F., and Souček, V., Clifford Algebra and Spinor-Valued Functions, Mathematics and its Applications Vol. 53 (Kluwer Academic Publishers Group, Dordrecht, 1992).

${ }^{5}$ De Schepper, N. and Sommen, F., "Closed form of the Fourier-Borel kernel in the framework of Clifford analysis," Results Math. 62(1-2), 181-202 (2012).

${ }^{6}$ Folland, G. B., Harmonic Analysis in Phase Space, Annals of Mathematics Studies Vol. 122 (Princeton University Press, Princeton, 1989).

${ }^{7}$ Gilbert, J. and Murray, M., Clifford Algebras and Dirac Operators in Harmonic Analysis, Cambridge Studies in Advanced Mathematics Vol. 26 (Cambridge University Press, 1991).

${ }^{8}$ Kirwin, W. D., Mourão, J., Nunes, J. P., and Qian, T., "Extending coherent state transforms to Clifford analysis," J. Math. Phys. 57(10), 103505 (2016).

${ }^{9}$ Mourão, J., Nunes, J. P., and Qian, T., "Coherent state transforms and the Weyl equation in Clifford analysis," J. Math. Phys. 58, 013503 (2017).

${ }^{10}$ Neretin, Y. A., Lectures on Gaussian Integral Operators and Classical Groups, EMS Series of Lectures in Mathematics (European Mathematical Society, 2011).

${ }^{11}$ Sabadini, I. and Sommen, F., “A Fourier-Borel transform for monogenic functionals on the Lie ball,” J. Math. Soc. Jpn. 68 , 1487-1504 (2016). 
${ }^{12}$ Segal, I. E., "Mathematical characterization of the physical vacuum for a linear Bose-Einstein field,” Ill. J. Math. 6, 500-523 (1962).

${ }^{13}$ Segal, I. E., "The Complex Wave Representation of the Free Boson Field," Topics in Functional Analysis, Adv. in Math. Suppl. Stud. (Academic Press, New York, London, 1978), Vol. 3, pp. 321-343 (essays dedicated to M. G. Krĕn on the occasion of his 70th birthday).

${ }^{14}$ Sommen, F., "Special functions in Clifford analysis and axial symmetry," J. Math. Anal. Appl. 130(1), 110-133 (1988).

${ }^{15}$ Sommen, F. and Jancewicz, B., "Explicit solutions of the inhomogeneous Dirac equation," J. Anal. Math. 71, 59-74 (1997).

${ }^{16}$ Voiculescu, D. V., Dykema, K. J., and Nica, A., Free Random Variables, Volume 1 of CRM Monograph Series (American Mathematical Society, Providence, RI, 1992).

${ }^{17}$ Zhu, K., Analysis on Fock Spaces, Graduate Texts in Mathematics Vol. 263 (Springer New York, 2012). 